\title{
Lateral subluxation of the atlanto-axial joint in rheumatoid arthritis
}

\author{
H. C. BURRY, ${ }^{1}$ J. M. TWEED, ${ }^{1}$ R. G. ROBINSON, ${ }^{3}$ AND R. HOWES ${ }^{2}$ \\ From the ${ }^{1}$ Hutt Hospital, Lower Hutt, and the ${ }^{2}$ Queen Elizabeth Hospital, Rotorua, New Zealand, \\ and the ${ }^{3}$ Royal North Shore Hospital, Sydney, Australia
}

SUMMARY Involvement of the cervical spine by rheumatoid disease is common, but lateral subluxation at the atlanto-axial level has not been recorded previously. The condition is due to asymmetrical erosion of the lateral atlanto-axial facet joint, and may be complicated by collapse of the lateral mass of the axis. The condition should be suspected in patients with rheumatoid arthritis (RA) who present with occipital, auricular, and/or facial pain.

Involvement of the cervical spine is a well-recognised and important feature of rheumatoid arthritis, its effects ranging from minor restriction of movement and discomfort to major neurological disability, and even death. The essential feature of this involvement is, as elsewhere, a synovitis which may erode, weaken, and destroy cartilage, bone, and ligamentous structures.

As might be expected, the most severely affected patients have usually suffered from a widespread destructive arthropathy and been seropositive.

Progressive erosive damage frequently involves the occipito-atlanto-axial region, the synovitis affecting the facetal joints as well as the synovial joints anterior and posterior to the odontoid process. Damage to the odontoid and to the transverse ligament results in an increased anteroposterior movement between the odontoid and the anterior arch of the atlas, which is easily seen radiologically. Vertical movement of the odontoid process into the foramen magnum may be seen when the facet joints at atlanto-axial level are destroyed. Not hitherto described is lateral subluxation of the atlas on the axis, and this communication records 3 such cases, and 2 others with unilateral erosive atlanto-axial facet joint involvement, which is considered to be an early manifestation.

\section{Case reports}

CASE 1 (Figs. 1, 2 and 3)

A 40-year old female, first presented at the Rheumatology Clinic, Queen Elizabeth Hospital in 1966 with a 2-year history of asymmetrical, predominantly

Accepted for publication January 20, 1978 small-joint polyarthritis. The ESR was $118 \mathrm{~mm} /$ hour; the Rose Waaler test was positive in a dilution of $1: 256$. Subcutaneous rheumatoid nodules were present. $X$-rays showed erosions in the small joints of the hands and feet.

In 1967 she complained of pain in the upper cervical region, but an $x$-ray of the cervical spine showed no significant abnormalities. Subsequently, her cervical pain became more severe, radiating upward over the occiput and behind the right ear. It was associated with pain behind the right eye. Her pain was aggravated by any movement of the neck, especially rotation, which was markedly restricted in range. The only neurological signs present were related to a bilateral carpal tunnel syndrome, and were relieved by surgical decompression of the wrists.

$X$-rays of the upper cervical spine repeated in 1974 showed erosion of the synovial joints between the lateral mass of the atlas and the axis on either side, with lateral subluxation. The medial aspect of the articular facet of the atlas appeared to butt against the base of the odontoid peg. Some erosions were also noted on the odontoid peg and, on cervical flexion, a gap of $4 \mathrm{~mm}$ was observed between the odontoid and anterior arch of the atlas. Her pain was relieved to some extent by corticosteroid and local anaesthetic infiltrations of the region of the suboccipital triangle and the use of a cervical collar.

CASE 2 (Fig. 4)

A 63-year old male, presented at the Rheumatology Clinic, Wellington Hospital, in 1965, with a 1-year history of widespread joint pain and swelling. He was observed to have a symmetrical peripheral arthritis with involvement also of the shoulders, and 


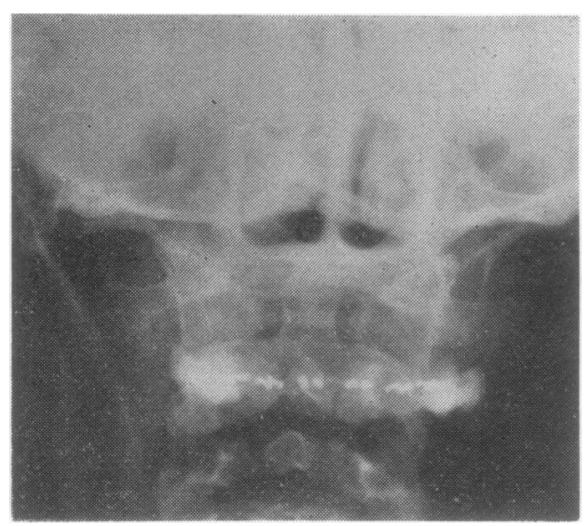

Fig. 1

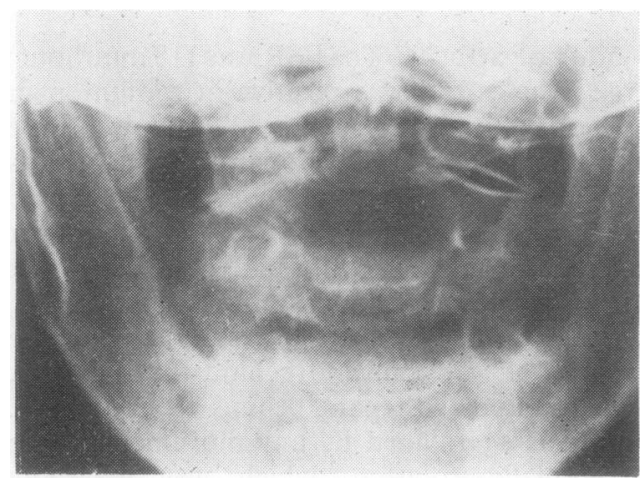

Fig. 2

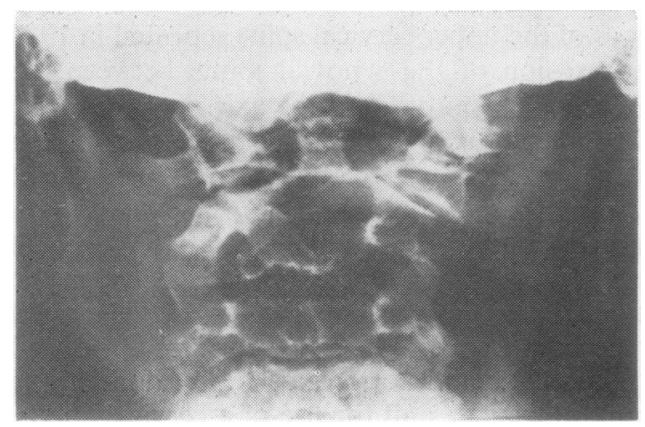

Fig. 3

Figs. 1, 2, and 3 Case 1. Slight narrowing of facet joints in 1967 (Fig. 1), proceeding to narrowing and irregularity with subchondral sclerosis and marginal erosions in 1972 (Fig. 2), and severe destructive arthropathy with lateral subluxation in 1974 (Fig. 3).

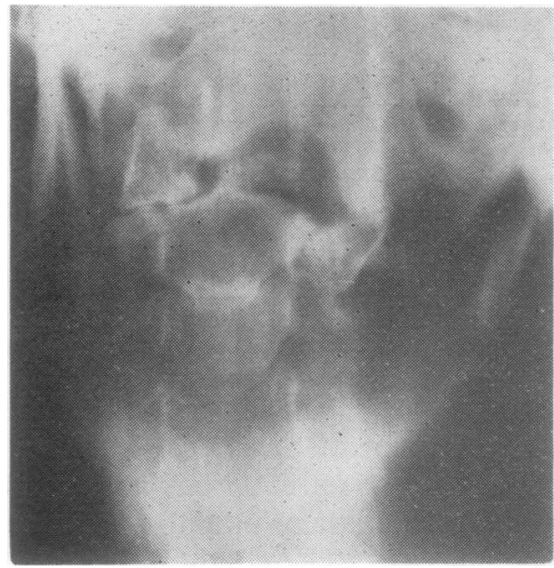

Fig. 4 Case 2. Gross destructive changes affecting both odontoid process largely destroyed, and atlanto-axial lateral facetal joints. The left lateral mass of the axis has collapsed allowing the atlas to tilt sharply to the left.

slight neck pain and stiffness. The ESR was elevate to $40 \mathrm{~mm} /$ hour; the Latex test for rheumatoid factor positive; DAT positive. 1:1024. The course of his disease was steadily progressive, with increas ing destruction of joints. Subcutaneous rheumato $f^{\infty}$ nodules appeared at the elbows and he later de oped episcleritis and scleromalacia.

By 1973, $x$-rays of the small joints of the hands and feet revealed arthritis multilans. At this time cervical pain had become more severe, and $x$-ray of the cervical spine revealed erosions of the odon $\vec{F}$ toid process, with a gap between the anterior archs of the atlas and the odontoid of $10 \mathrm{~mm}$ on fulb forward flexion. Erosive changes were also noted in the apophyseal joints in the mid-cervical region.

In 1975, following a fall, his cervical pain an $\overline{\text { }}$ stiffness became much more severe so that he was more or less confined to bed. The head was held in a position of flexion to the left, movements being restricted to forward flexion $60^{\circ}$, rotation $30^{\circ}$ to either side. No extension or side flexion to right of left was possible, and there was considerable pain on all movements. Marked tenderness was presen? in the region of the suboccipital triangle and overn the lateral mass of the atlas on the left side. No neurological abnormalities could be demonstrated although the scalp felt slightly tender over the occiput

$X$-rays now revealed that the articular facet of the axis on the left side had been completely erodede allowing the lateral mass of the atlas to sublux, while the articular facet on the right lateral mass of the atlas was impinging upon the base of the odontoid The odontoid itself showed massive erosions. 


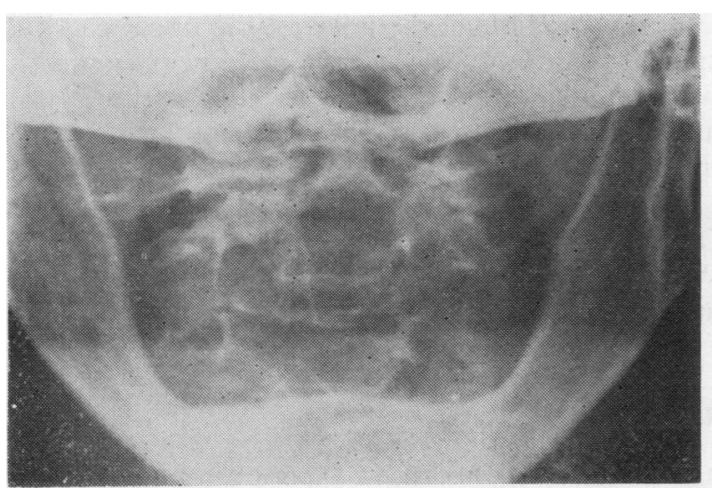

Fig. 5 Case 3. Severe destructive changes in lateral atlanto-axial joints with descent of atlas to the left.

CASE 3 (Fig. 5)

A 57-year old female, with a 14-year history of RA had experienced progressive loss of movement and pain in the cervical region. Total range of rotation was reduced to $70^{\circ}$. She had not experienced headaches, other than an occasional attack of classical migraine. She was seropositive, Rose Waaler 1:80, had subcutaneous nodules, arteritic nail-fold lesions, and severe articular erosions. $X$-rays of the cervical spine revealed destructive changes in the left lateral atlanto-axial joint, with descent of the lateral mass of the atlas. There was also an increased gap on flexion between the anterior arch of the atlas and odontoid peg.

\section{CASE 4 (Fig. 6)}

A 61-year old female had suffered from RA since the age of 20. She complained of pain in the upper cervical region, radiating to the mastoid region. A Rose Waaler test was positive $1: 512$ and there were many erosions. An $x$-ray of the cervical spine

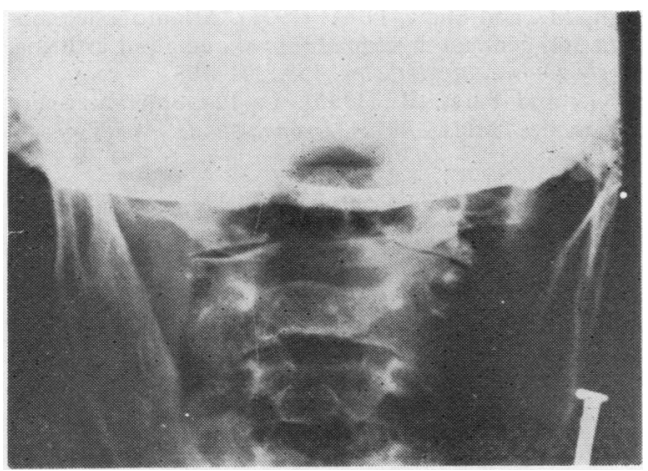

Fig. 6 Case 4. Unilateral atlanto-axial joint destruction without displacement. confirmed that there was unilateral marked jointspace narrowing of the lateral atlanto-axial joint.

\section{CASE 5}

A 74-year old female had suffered from RA for 30 years. A Rose Waaler test was positive 1:256, and there were numerous joint erosions. She presented with pain in the occipital region and limitation of cervical movements. An $x$-ray showed almost complete obliteration of the left lateral atlantoaxial joint, but no displacement.

\section{Discussion}

Involvement of the cervical spine in rheumatoid arthritis has been the subject of numerous publications since the first definite description of vertical subluxation in this area in 1951 by Davis and Markley. Abnormalities which have been described include atlanto-axial subluxation in anteroposterior as well as vertical planes (Vignon and Patet, 1955), subluxation below the atlanto-axial joint (Kornblum et al., 1952), apophyseal joint fusion and vertebral collapse (Baggenstoss et al., 1952), end-plate erosions (Meikle and Wilkinson, 1971), disc-space narrowing without osteophytosis (Ball, 1958), and erosion of the spinous processes and ankylosis (Seaman and Wells, 1961). A number of excellent reviews of the subject have been published (Conlon et al., 1966; Stevens et al., 1971; Smith et al., 1972).

Clinically, arthritis of the lateral atlanto-axial joints is likely to present as pain and/or stiffness in the upper cervical region, with painful restriction of rotation of the cervical spine. The pain may be referred to the occipital and auricular areas, and sometimes to the orbit. As subluxation occurs, inclination of the head toward the side of the subluxation is usual, and rotation, which takes place to a very large extent at atlanto-axial level, becomes very severely restricted.

Serial radiographs show that the sequence of events leading to lateral subluxation of the atlantoaxial joint is joint-space narrowing (Figs. 1-6) presumably due to articular cartilage erosion; erosion of subchondral bone; disruption of the joint capsule allowing lateral shift, and, in the severest case, collapse of the lateral mass, allowing the atlas to tilt sharply laterally.

No neurological abnormalities could be demonstrated to indicate the presence of either myelopathy or nerve-root compression, nor were there any signs or symptoms of vertebral artery obstruction. The latter is surprising in view of the close proximity of the artery to the vertebral lesion.

Lateral subluxation of the atlanto-axial joint only 
becomes evident when an AP film of the atlantoaxial region is taken through the open mouth. This view is not normally included in radiography of the cervical spine, and this may account for the absence of any reference to lateral subluxation in the literature related to rheumatoid arthritis and its complications. The condition may not be as rare as might be supposed. Certainly, upper cervical pain radiating to the occiput is fairly common in patients with rheumatoid arthritis (Coutts, 1934; Laurie and Stewart, 1961; Dugan et al., 1962), although noted in only 2 of the patients studied by Stevens $e t$ al., (1971). When present, it is frequently intractable, defying the usual techniques of therapy. The reference of pain to the occiput and behind the pinna would certainly be consistent with irritation of the second cervical nerve root. This root emerges from below the posterior arch of the atlas, to enter the suboccipital triangle lying in close proximity to the vertebral artery. With lateral displacement of the atlanto-axial joints, one could easily envisage that the consequent encroachment upon an already restricted space would produce pressure upon the nerve root. Thus, symptoms over the occiput and in the region of distribution of the great auricular nerve could be expected. Reference of pain to the region of the orbit is more difficult to explain. Similar symptoms are seen not uncommonly in patients with cervical spondylosis accompanied by tension states, and this condition is sometimes referred to as occipital neuralgia. The predominantly unilateral nature of the condition, and the presence of pain in the region of the eye, often suggests an erroneous diagnosis of migraine. One possible explanation of the nature and quality of the pain might be that the second cervical root communicates via the ansa cervicalis with the trigeminal nerve, so that projection of symptoms to the face might be anticipated. In the two cases described, no neurological abnormalities were noted and, in particular there was no evidence of myelopathy in either case. This is probably due to the wide diameter of the cervical canal in this region, and the comparatively small amount of lateral movement possible, even in the presence of gross destructive changes, as in case 2.
Further radiological studies of the upper cervical spine by means of AP views through the open mout will be required in patients presenting with cervicat and cranial pain to determine the true incidence of this interesting manifestation of rheumatoid disease?

Our thanks are due to Drs B. L. J. Treadwell, Weston, J. Kirk, and R. Gibson for their assistance in preparing this paper.

\section{References}

Baggenstoss, A. H., Bickell, W. H., and Ward, L. E. (1952) Rheumatoid granulomatous nodules as destructive lesion of vertebrae. Journal of Bone and Joint Surgery, 34A, 601-609.

Ball, J. (1958). Pathology of the rheumatoid cervical spines Lancet, 1, 86.

Conlon, P. W., Isdale, I. C., and Rose, B. G. (1966 Rheumatoid arthritis of the cervical spine. Annals of thg Rheumatic Diseases, 25, 120-126.

Coutts, M. B. (1934). Atlanto-epistropheal subluxations? Archives of Surgery, 29, 297-311.

Davis, F. W., Jr., and Markley, H. E. (1951). Rheumatoi $\Phi^{\circ}$ arthritis with death from medullary compression. Annak of Internal Medicine, 35, 451-454.

Dugan, M. C., Locke, S., and Gallagher, J. R. (1962) Occipital neuralgia in adolescents and young adults. New England Journal of Medicine, 267, 1166-1172.

Kornblum, D., Clayton, M. L., and Nash, H. H. (1932) Nontraumatic cervical dislocations in rheumatoid sponfylitis. Journal of the American Medical Association, 431-435.

Lourie, E., and Stewart, W. A. (1961). Spontaneous atlanto axial dislocation. New England Journal of Medicine, 265. 677-681.

Meikle, J. A. K., and Wilkinson, M. (1971). Rheumatoi\& involvement of the cervical spine. Annals of the Rheumat Diseases, 30, 154-161.

Seaman, W. B., and Wells, J. (1961). Destructive lesions of the vertebral bodies in rheumatoid disease. Americam Journal of Roentgenology, 86, 241-250.

Smith, P. H., Benn, R. T., and Sharp, J. (1972). Naturats history of rheumatoid cervical subluxations. Annals of the Rheumatic Diseases, 31, 431-439.

Stevens, J. C., Cartlidge, N. E. F., Saunders, M., Appleb产 A., Hall, M., and Shaw, D. A. (1971). Atlanto-axial subluxation and cervical myelopathy in rheumatoid arthritio Quarterly Journal of Medicine, 159, 391-408.

Vignon, G., and Patet, R. (1955). La luxation antérieure spontanée de l'atlas. Revue Lyonnaise de Médicine, 693-702. 